BOOKS

\title{
Good advice, if you can follow it
}

Previously published at www.cmaj.ca

\author{
A Bitter Pill: How the Medical System is \\ Failing the Elderly \\ John Sloan \\ Greystone Books; 2009.
}

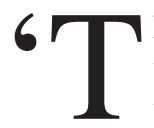

he medical system is set up to help people, but it does

the opposite for most of the people I look after," writes Dr. John Sloan, a family physician who restricts his practice to housebound, mainly older frail persons. These are harsh words indeed, but Sloan offers compelling evidence of their veracity.

$\mathrm{He}$ is talking about the frail elderly, "people with a lot of health problems, who are dependent on others for daily activities, for whom function and a functional approach to [a medical] crisis are important, for whom comfort is a priority and who are very different from one another ... [and are] on quite a bit of medication." In scenarios well known at least to geriatricians and many experienced family doctors, Sloan describes how older frail persons with multiple pathology are inappropriately treated by a system which assumes that diseases must be addressed individually. Each malady is treated in accordance with established clinical guidelines,

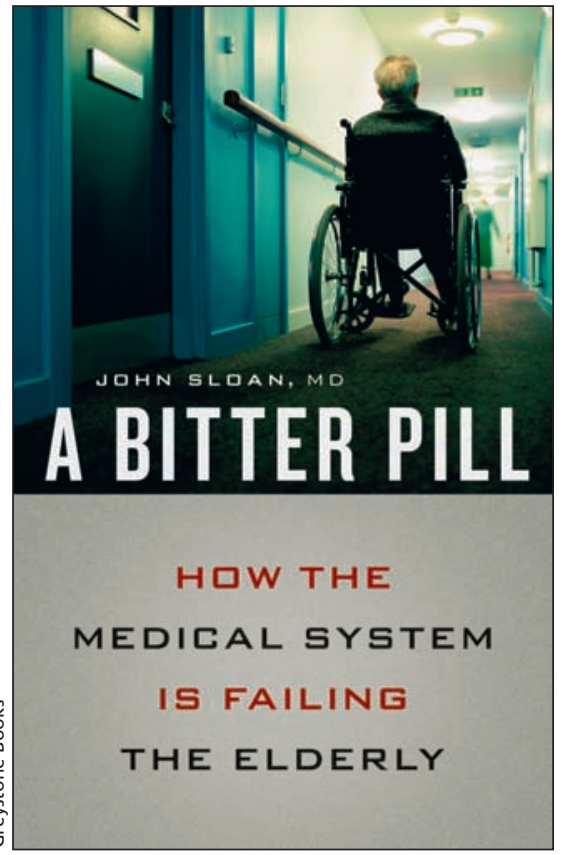

cally, although Sloan doesn't address the situation of the frail elderly in the developing world, according to his argument, these people should not do so badly given a general lack of high technology.)

Interestingly, despite its medicaloid title, this book is not directed to physicians, but rather to caregivers and to the frail elderly. The author's solutions are simple and perhaps sometimes a bit simplistic. Take, for example, this recommendation to caregivers: “... after

\section{The author's solutions are simple and sometimes a bit simplistic.}

which address that individual problem, but do not take into account the myriad other pathologies such people can carry. But the system, including drug companies, academic physicians - for whom he seems often to have little time - and many other actors such as government and insurance agencies, seem to almost actively conspire against the frail elder.

In my opinion, Sloan is correct in his claims and the problem is not restricted to Canada, but can be observed anywhere in the industrialized world. (Ironi- deciding to get rid of a prevention-andrescue approach to care ... get responsibility for an old person into your own hands, away from the experts and out of institutions. ... What we want is no drugs that don't [sic] make the person feel better and no hospital unless there is a clear goal that can't be met any other way." He goes on to give the caregiver a good old metaphorical clap on the back: "Just decide, and then stick to that decision as much as possible." (Emphasis in the original.)
Good advice - if you can follow it. And there to me is the rub. For a caregiver to actually take the course outlined in the book, he or she would have to be reasonably educated with significant resources - financial, familial, physical and above all emotional - to carry this heavy load. As well, this ideal household would still need ready access to a kindly physician such as Sloan with an abundant expression of the three As (ability, affability and availability). At least as far as I know, such doctors are not exactly commonplace.

The clear mismatch between the true medical needs of the frail elderly person and the often juggernaut-like qualities of modern medicine is sadly pretty well known. The question of what to do about it is less well formulated and it certainly needs more than a vigorous and willing caregiver. The problem that Sloan describes so eloquently can only be properly addressed by a combination of changes in medical education, in attitudes of doctors and the proper organization of services for the housebound elderly, such as the SIPA project (a system of integrated services for the frail elderly) in Quebec with which I had the privilege of being associated. (Unfortunately, while this program has been investigated, evaluated and published, it has not yet been implemented).

We can derive some hope by observing the development of the hospice/ palliative care movement, which has done so much to modify our approach to the end-stage cancer patient.

This book, if read by physicians and health care authorities, should strengthen their resolve to make the appropriate changes. And we had all better act pretty quickly as so many of us will soon be reaching the age where frailty, like cancer, can strike any of us.

\section{A. Mark Clarfield MD \\ Department of Geriatrics \\ Soroka Hospital \\ Ben-Gurion University of the Negev \\ Beer-Sheva, Israel}

